


\title{
CENTER VORTICES, MAGNETIC CONDENSATE AND CONFINEMENT IN A SIMPLE GAUGE SYSTEM
}

\author{
F. GLIOZZI AND M. PANERO \\ Dipartimento di Fisica Teorica dell'Università di Torino, \\ Istituto Nazionale di Fisica Nucleare, Sezione di Torino, \\ via P. Giuria 1, I 10125 Torino, Italy \\ AND \\ P. PROVERO \\ Dipartimento di Scienze e Tecnologie Avanzate \\ Università del Piemonte Orientale, Alessandria, \\ Istituto Nazionale di Fisica Nucleare, \\ gruppo collegato di Alessandria, I 15100 Alessandria, Italy
}

\begin{abstract}
The confining mechanisms of 't Hooft and Mandelstam have a simple microscopic realization in $3 \mathrm{D} \mathbb{Z}_{2}$ gauge theory: the center vortex and the magnetic monopole condensation are associated, in the set of configurations contributing to the confining phase, to the presence of two kinds of infinite clusters. These generate the area law of the large Wilson loops and the universal finite size effects produced by the quantum fluctuations of the bosonic string describing the infrared behavior of the flux tube.
\end{abstract}

\section{Introduction}

Two kinds of topological excitations were proposed by 't Hooft as possible composite fields condensing in the disordered phase of gauge systems to produce confinement: magnetic monopoles [1] and center vortices [2].

Condensation of magnetic monopoles implies dual superconductivity of the vacuum and gives the well-known physical picture of confinement in terms of dual Abrikosov vortices which describe the flux tubes.

Center vortices are string-like structures which are created by gauge transformations with a non-trivial homotopy. Their condensation produces a very efficient disordering of the gauge configurations. 
In the last few years, many lattice studies have been made on these two subjects in the last few years (see for instance the two review talks of Di Giacomo [3] and Olejník [4] at this Conference).

These two kinds of confining mechanisms have a particularly simple implementation in the $3 \mathrm{D} \mathbb{Z}_{2}$ gauge model, where it is possible to study this phenomenon not only in terms of the effective degrees of freedom, using for instance the underlying string description of the infrared behavior of the flux tube, but also at a microscopic level, i.e. in terms of $\mathbb{Z}_{2}$ lattice configurations: it is possible to pick out the degrees of freedom which are relevant for the infrared dynamics of the confining phase.

It turns out that the condensation of the above-mentioned topological excitations manifests itself at the microscopic level with the appearance of two (complementary) infinite clusters of links in the dual lattice. Both produce not only area law decay of the vacuum expectation value of large Wilson loops, but also the universal finite size effects which are predicted by the free bosonic string picture of the flux tube.

\section{Monopole condensation}

The three-dimensional $\mathbb{Z}_{2}$ gauge model on a cubic lattice is defined by the action

$$
S(\beta)=-\beta \sum_{\square} U_{\square} \quad, U_{\square}=\prod_{\ell \in \square} U_{\ell}
$$

where $U_{\ell}= \pm 1$ is the $\mathbb{Z}_{2}$ gauge field associated to the links $\ell$ of the lattice $\Lambda$. The magnetic monopoles live in the dual lattice $\tilde{\Lambda}$. In order to create a monopole in a site $\tilde{x} \in \tilde{\Lambda}$ corresponding to the center of an elementary cube of $\Lambda$ it is sufficient to draw an arbitrary, continuous line $\gamma(\tilde{x})$ joining $\tilde{x}$ to $\infty$ and flipping the sign of the coupling of the plaquettes crossed by $\gamma$. This flipping is generated by the non-local operator

$$
\Psi_{\gamma}(\tilde{x})=\exp \left(-2 \beta \sum_{\square \in \gamma} U_{\square}\right)
$$

As a consequence, the flux across any closed surface around $\tilde{x}$ is equal to -1 : this monopole field $\Psi_{\gamma}(\tilde{x})$ creates one unity of $\mathbb{Z}_{2}$ flux. Thus the monopole is associated to a point-like topological defect in the ensemble of the gauge configurations.

Monopole condensation occurs when $\Psi$ acquires a vacuum expectation value different from zero:

$$
\text { monopole condensation } \Longleftrightarrow\langle\Psi\rangle \neq 0 \text {. }
$$

Why this condensation should imply confinement? A useful piece of information comes from the Kramers- Wannier duality. This transformation 
maps the $\mathbb{Z}_{2}$ gauge theory into the Ising model described by the Hamiltonian

$$
H(\beta)=-\tilde{\beta} \sum_{\langle\tilde{x} \tilde{y}\rangle} \sigma_{\tilde{x}} \sigma_{\tilde{y}} \quad, \tilde{\beta}=-\frac{1}{2} \log \tanh \beta
$$

with $\langle\tilde{x} \tilde{y}\rangle$ ranging over the links of $\tilde{\Lambda}$ and the spin variable $\sigma_{\tilde{x}}$ taking the values \pm 1 . As a consequence one can easily show that

$$
\left\langle\Psi_{\gamma}(\tilde{x})\right\rangle=\left\langle\sigma_{\tilde{x}}\right\rangle_{I \operatorname{sing}}
$$

Thus the magnetic condensation is associated with the spontaneous $\mathbb{Z}_{2}$ symmetry breaking of the Ising model.

The same transformation maps the Wilson loop $W(C)$ associated to any closed curve $C \in \Lambda$ into the corresponding 't Hooft loop $\widetilde{W}(C)$ of the Ising model:

$$
\langle W(C)\rangle=\langle\widetilde{W}(C)\rangle_{I s i n g}
$$

with

$$
\widetilde{W}(C)=\exp \left(-2 \tilde{\beta} \sum_{\langle i j\rangle \in \Sigma} \sigma_{i} \sigma_{j}\right), \partial \Sigma=C
$$

where $\Sigma$ is an arbitrary surface bounded by $C$.

$\widetilde{W}(C)$ creates an elementary $\mathbb{Z}_{2}$ flux along $C$ which manifests itself as a topological defect or a frustration in the ensemble of the Ising configurations: the product of the link variables along any loop $\widetilde{C} \in \widetilde{\Lambda}$ having an odd linking number with $C$ is equal -1 .

Confinement requires that $\langle\widetilde{W}(C)\rangle_{\text {Ising }}$ should decay with an area law in the ordered phase of the Ising model. To understand this property at a microscopic level it is convenient to resort to the Fortuin Kasteleyn (FK) random cluster representation of the model:

$$
Z=\sum_{\{\sigma\}} e^{-H(\beta)}=\sum_{G \subseteq \Lambda} v^{b_{G}} 2^{c_{G}},
$$

where $v=e^{\tilde{\beta}}-1$, the summation is over all spanning subgraphs $G \subseteq \Lambda$. $b_{G}$ is the number of links of $G$, called active bonds, and $c_{G}$ is the number of connected components, called FK clusters. The ordered phase is characterised by the presence of an infinite, percolating FK cluster.

When there are frustrations in the system (for instance those generated by $\widetilde{W}(C))$ the summation in Eq.7 is constrained. Only those FK clusters are allowed for which no closed path within the cluster is linked to an elementary $\mathbb{Z}_{2}$ flux [9]. This is a microscopic realization of a sort of dual Meissner effect: the FK clusters behave like pieces of dual superconducting matter of 


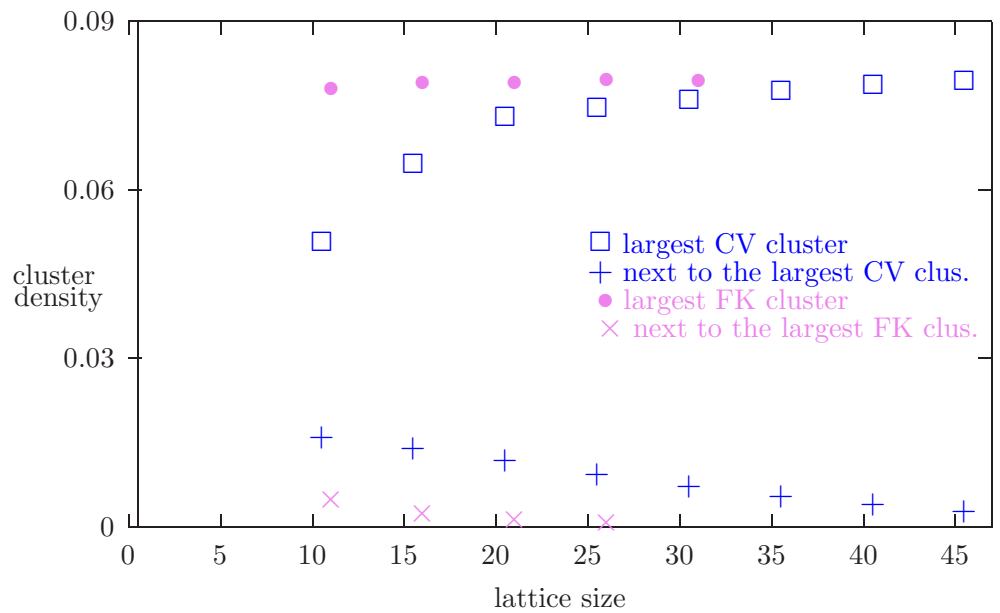

Figure 1. Density of the largest center vortex (squares) and FK (circles) clusters. The densities of the second largest clusters of the two types are also shown.

type I and no $\mathbb{Z}_{2}$ flux can go through them. Introducing a projector $\varpi_{C}(G)$ on the configurations $G \subseteq \Lambda$ which takes the value 0 whenever there is a FK cluster (at least) linked to $C$, and the value 1 in all the other cases, yields the very useful identity

$$
\langle W(C)\rangle=\left\langle\varpi_{C}\right\rangle_{I s i n g}=\frac{\text { number of allowed config. }}{\text { total number of config. }} .
$$

Assume that there are only FK clusters of finite size ( this is the case of the symmetric phase). If $C$ is much larger than the mean size of the clusters, the configurations contributing to $W(C)$, i.e. those with $\varpi_{C}(G)=1$, are characterised by the fact that there is no cluster linked to $C$. The weight of this class of configurations, when compared with the total ensemble $G \subseteq \Lambda$ is clearly suppressed by a factor $e^{-\alpha p(C)}$, where $p(C)$ is the length of $C$. Thus the Wilson loop obeys a perimeter law in this phase. Conversely we can argue that a decay of large Wilson loops with an area law implies the presence of an infinite FK cluster. Quite remarkably, this kind of reasoning, based only on concepts well defined at the microscopic level, leads to the very important, unavoidable, conclusion that confinement requires monopole condensation. This is just the reverse of the usual 't Hooft confinement criterion, which can be justified using plausibility arguments based on the effective description in terms of (dual) Abrikosov vortices.

The above arguments lead us to conjecture that the infinite FK cluster encodes the most relevant part of the infrared dynamics of the confined phase. More precisely, starting from the the asymptotic form of the vacuum 
expectation value of a large rectangular Wilson loop

$$
\langle W(R, T)\rangle=\exp (-\sigma R T+p(R+T)+c)\left[\frac{\sqrt{R}}{\eta(\tau)}\right]^{\frac{1}{2}}, \tau=i \frac{T}{R},
$$

where $\eta(\tau)$ is the Dedekind function and the term within square brackets is the universal contribution due to the quantum fluctuations of the flux tube, it is easy to prove a sort of no-renormalization theorem: the vacuum expectation value of $W(R, T)$ in the ensemble of configurations obtained by those in thermal equilibrium erasing all the finite FK clusters has exactly the same functional form of Eq.9 and with the same value of $\sigma$. The argument goes as follows: let $N$ be the total number of configurations and $N_{1}$ those compatible with $C$. We have $\langle W(C)\rangle=\frac{N_{1}}{N}$. When the finite FK clusters are eliminated, the number $N_{1}^{\prime}$ of compatible configurations increases $\left(N_{1}^{\prime}>N_{1}\right)$ because some graph with $\varpi_{C}(G)=0$ is promoted to a compatible one. The perimeter law decay produced by the finite FK clusters can now be used to get $N_{1}=e^{-\alpha p(C)} N_{1}^{\prime}$, which completes the proof.

The transformation $N_{1} \rightarrow N_{1}^{\prime}$ reduces drastically the noise in the evaluating of large Wilson loops and it has been used also in related models to study the universal shape effects related to the quantum fluctuations of the flux tube [5].

\section{Clusters of center vortices}

Center vortices in $Z_{2}$ gauge model are constructed by assigning a vortex line in the dual lattice to each frustrated plaquette $\left(\right.$ i.e. $\left.U_{\square}=-1\right)$ in the direct lattice. Since the product of the six plaquettes forming a cube is constrained to be equal to 1 , the resulting graph of center vortices (CV) in $\widetilde{\Lambda}$ has even coordination number. This has to be contrasted with the FK clusters of the previous section which are arbitrary subgraphs of $\widetilde{\Lambda}$.

The value of a Wilson loop $W(C)$ in a given configuration is \pm 1 according to the number, modulo 2, of frustrated plaquettes of an arbitrary surface $\Sigma$ bounded by the loop $(\partial \Sigma=C)$, or, in the center vortex language, to the number, modulo 2 , of vortex lines that are linked to the loop. The role of center vortices in confinement in this model is in a way trivial, since removal of all center vortices from $\mathbb{Z}_{2}$ gauge theory configurations simply removes all the dynamics by transforming every configuration into the trivial vacuum. Therefore center vortices are, in this sense, trivially responsible not only for confinement, but for the whole dynamics of the model.

We are interested to a subtler issue, namely the effect of the finite $\mathrm{CV}$ clusters on confinement [6]. Also in this case it is easy to prove that

confinement requires an infinite $\mathrm{CV}$ vortex, however it is no longer possible 
to prove the no-renormalization theorem of the FK formalism, hence there is no reason to believe that the string tension does not change under the transformation erasing the finite CV clusters.

It is straightforward to verify numerically that in the confining phase of $\mathbb{Z}_{2}$ gauge system there is no ambiguity in finding a cluster of center vortices whose size scales linearly with the lattice volume for large enough lattices (see Fig.1), while in the deconfined phase we found that the density of the largest cluster decreases rapidly with the volume.

It has to be noted that the presence of the infinite cluster does not necessarily imply a percolation property of the central vortices. Confinement requires merely the presence of an infinite cluster. In the FK representation this requirement is sufficient to assure percolation of the infinite FK cluster, while there are regions in the confining phase where the infinite CV cluster does not percolate, perhaps because of the even coordination constraint.

To study more carefully the relationship between the presence of the infinite cluster and the value string tension, we chose [10] to simulate the model at $\beta=0.74883$, which is well inside the scaling region, and for which the value of the string tension is known with high precision from simulations of the dual model, that is the 3D (spin) Ising model $[7,8] \sigma=0.01473(10)$

First, we verified the existence of an "infinite" cluster of vortex lines: for each configuration, we selected the largest connected component of the graph defined by the center vortices, and verified that the size of such component grows linearly with the volume of the lattice.

The results of this analysis are shown in Fig. 1, where the size of the largest CV cluster divided by the lattice volume is shown to approach a constant for large lattices. The size of the second largest cluster is also shown: its size relative to the lattice volume tends to zero and the identification of the "infinite" cluster is unambiguous. For comparison, also the corresponding FK cluster densities are reported.

To test the relevance of the largest cluster of center vortices, we proceeded as follows: first, we modified each configuration in the Monte Carlo ensemble by eliminating all the vortices not belonging to the largest cluster, and second, by eliminating, instead, the largest cluster only. The qualitative picture described above suggests that a non-zero string tension will be found in the first case but not in the second.

An efficient method to extract the string tension from Wilson loop data generated by Monte Carlo simulations, which takes into account the string fluctuation contribution, was introduced in Ref. [11]. One defines the ratio of the expectation values of rectangular Wilson loops with the same perimeter:

$$
r(R, n)=\frac{\langle W(R+n, R-n)\rangle}{\langle W(R, R)\rangle}
$$


Full configurations

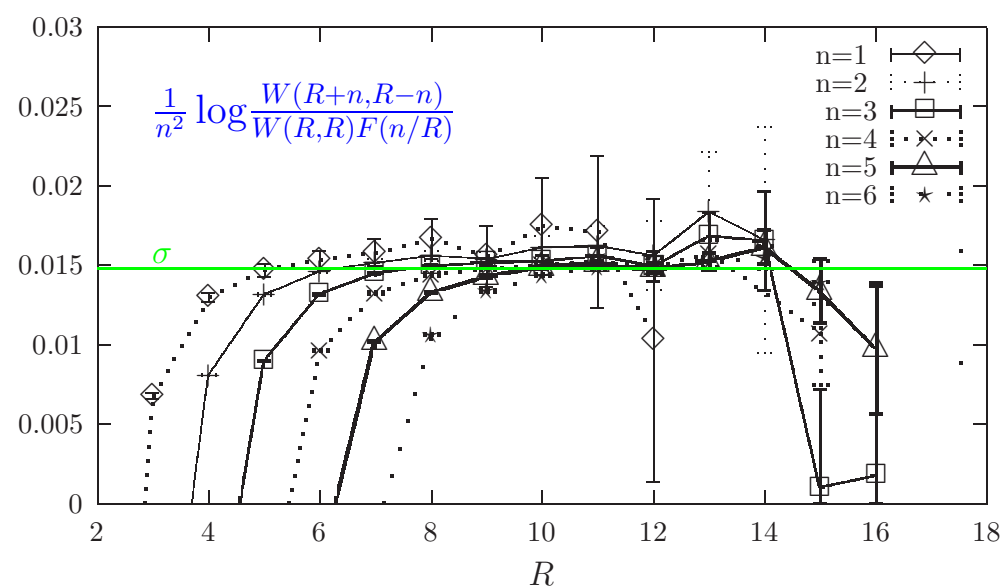

(a)

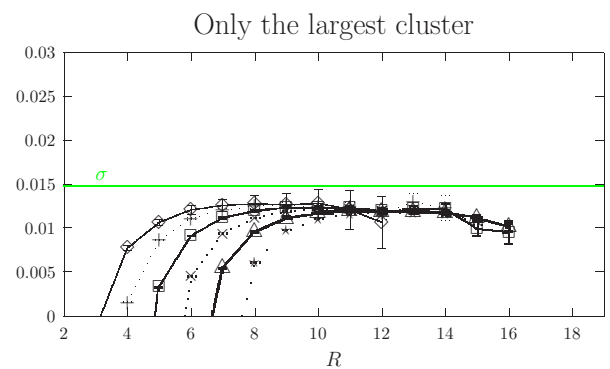

(b)

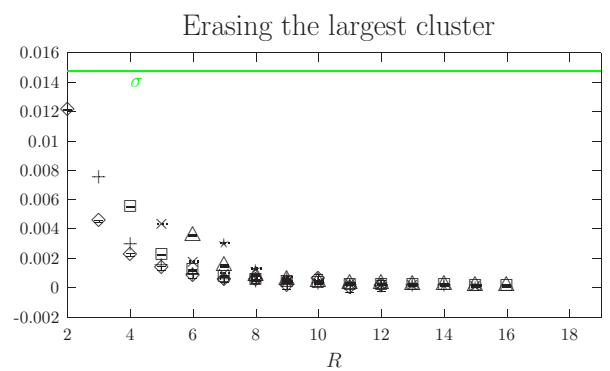

(c)

Figure 2. (a) $\sigma_{\text {eff }}(R, n)$ as a function of $R$ for $n=2,3,4,5,6$ for the original configurations. (b) Th same quantity for the configurations where only the largest cluster of center vortices has been left. (c) The effect of removing only the largest cluster.

Using the asymptotic expansion defined in Eq.9 we get

$$
r(R, n) \sim \exp \left(\sigma n^{2}\right) F(n / R)
$$

where $F(t)=\sqrt{\eta(i) \sqrt{1-t} / \eta\left(i \frac{1+t}{1-t}\right)}$, so that one can define the following quantity

$$
\sigma_{\text {eff }}(R, n)=\frac{1}{n^{2}} \log \left(\frac{r(R, n)}{F(n / R)}\right)
$$

which approaches the string tension for large enough $R$. The results of our simulations are reported in Fig.2

In conclusion, our results confirm the picture of confinement as due to the existence of an infinite cluster of center vortices: our choice of the $\mathbb{Z}_{2}$ 
gauge theory allows us to bypass all the problems related to the gaugefixing and center projection that one encounters when studying the same issue in $S U(N)$ gauge theories. Two important new facts emerge from our study:

While the largest center vortex is responsible for confinement, since its removal from the configurations makes the string tension vanish, the string tension measured from configurations in which all the other clusters have been removed does not reproduce the full string tension of the original theory. Therefore small clusters of vortices, while unable by themselves to disorder the system enough to produce confinement, do give a finite contribution to the string tension of the full theory. This has to be contrasted with the FK cluster formulation, where the infinite cluster account for the whole string tension, because of a no-renormalization theorem.

The quantum fluctuations of the flux tube survive the elimination of the small CV or FK clusters: the Wilson loop after deletion of all the small clusters show the same shape dependence as the ones of the full theory, which can be explained as originating by the fluctuations of a free bosonic string.

\section{References}

1. 't Hooft,G (1981) Topology Of The Gauge Condition And New Confinement Phases In Nonabelian Gauge Theories, Nucl. Phys. B 190, 455.

2. 't Hooft, G. (1978) On the phase transition towards permanent quark confinement, Nucl. Phys. B B138, 1

3. Di Giacomo,A., (2002) Color confinement and dual superconductivity: an update, arXiv:hep-lat/0204032.

4. Faber,M., Greensite,J. and Olejnik,S., (2002) Status of center dominance in various center gauges, arXiv:hep-lat/0204020.

5. Gliozzi, F. and Rago, A., (2001) String breaking mechanisms induced by magnetic and electric condensates, Nucl. Phys. Proc. Suppl. 106, 682 [arXiv:hep-lat/0110064].

6. Engelhardt,M., Langfeld,K.,Reinhardt, H. and Tennert,O., (2000) Deconfinement in SU(2) Yang-Mills theory as a center vortex percolation transition, Phys. Rev. D 61, 054504 [arXiv:hep-lat/9904004].

7. Hasenbusch, M., and Pinn, K., (1992) Surface tension, surface stiffness, and surface width of the three-dimensional Ising model on a cubic lattice, Physica A 192, 342 [arXiv:hep-lat/9209013].

8. Caselle,M. Fiore,R.,Gliozzi,F., Hasenbusch,M.,Pinn, K. and Vinti,S. (1994) Rough interfaces beyond the Gaussian approximation, Nucl. Phys. B 432, 590 [arXiv:heplat/9407002].

9. Caselle, M. and Gliozzi, F. (2000) Thermal Operators in Ising Percolation, J. Phys. A 33,2333 [arXiv:cond-mat/9905234].

10. Gliozzi,F.,Panero,M. and Provero,P. (2002) Large center vortices and confinement in $3 \mathrm{D} \mathrm{Z}(2)$ gauge theory, arXiv:hep-lat/0204030.

11. Caselle,M.,Fiore,R.,Gliozzi,F.,Hasenbusch,M. and Provero,P.(1997) String effects in the Wilson loop: A high precision numerical test, Nucl. Phys. B 486, 245 [arXiv:heplat/9609041]. 